\title{
La Educación en Tiempo del Covid-19 Herramientas TIC: EI Nuevo Rol Docente en el Fortalecimiento del Proceso Enseñanza Aprendizaje de las Prácticas Educativa Innovadoras
}

\author{
Covid-19 Education in Time ICT Tools: The New Teaching Role in Strengthening the \\ Teaching Process of Innovative Educational Practices
}

Carlos Henry Sandoval

\section{ఠ} EDICIÓN: \#E-CIVTAC

Recibido: 3/junio/2020

Aceptado: $9 /$ julio/2020

Publicado: 25/septiembre/2020

${ }^{1}$ Colombia

\section{IIIIInstitución \\ ${ }^{1}$ Corporación Universitaria minuto de Dios}

\section{Correo Eletrónico}

${ }^{1}$ carlos.sandoval@uniminuto.edu

\section{(D) ORCID}

'https://orcid.org/0000-0003-4834-2037

\section{Citar así: GCAPA / IEEE}

Sandoval, C. (2020). La Educación en Tiempo del Covid-19 Herramientas TIC: El Nuevo Rol Docente en el Fortalecimiento del Proceso Enseñanza Aprendizaje de las Prácticas Educativa Innovadoras. Revista TecnológicaEducativa Docentes 2.0, 9(2), 24-31. https://doi.org/10.37843/rted.v9i2.138

C. Sandoval, "La Educación en Tiempo del Covid-19 Herramientas TIC: El Nuevo Rol Docente en el Fortalecimiento del Proceso Enseñanza Aprendizaje de las Prácticas Educativa Innovadoras", RTED, vol. 9, n. ${ }^{\circ}$ 2, pp. 24-31, sep. 2020.

\section{Resumen}

La Organización Mundial de la Salud (OMS), ha declarado al COVID-19 como pandemia por su rapidez de propagación, el sector educativo no escapa a ello. Los colegios, con plataformas digitales, tienen opción de flexibilizar procesos pedagógicos en los estudiantes a partir de la articulación de aplicaciones web con el fin de continuar con el servicio educativo. El presente estudio tuvo como objetivo analizar la integración de las herramientas TIC como estrategia didáctica en el aprendizaje colaborativo en los espacios de formación académica como parte del replanteamiento del ejercicio docente. Para el trabajo de investigación se utilizó el enfoque mixto. El instrumento empleado fue el cuestionario, lo que permitió diagnosticar sobre el manejo y uso de las herramientas TIC con el fin de poder responder a las necesidades individuales y colectivas planteadas por los educadores y las directivas de las instituciones educativas. En los resultados obtenidos se evidenció que el educador debió asumir un nuevo rol mediado por las TIC frente al aislamiento preventivo obligatorio. Otro hallazgo fue el fortalecimiento del uso de las TIC en el contexto virtual de las clases generada por los educadores, dado un nuevo impulso al proceso formativo a partir de experiencias significativas innovadoras. Se concluye que la pandemia generó una disrupción en el sistema educativo, por tal razón, las directivas de las instituciones educativas deben tener como alternativas de aprendizaje efectivo el modelo de educación en modalidad virtual como valor agregado a su propuesta educativa en respuesta al nuevo contexto posCOVID-19.

Palabras clave: Herramientas TIC, COVID-19, estrategia didáctica, innovación educativa.

\section{Abstract}

The World Health Organization (WHO) has declared COVID-19 a pandemic due to its speed of spread, the education sector is not immune to it. Schools, with digital platforms, have the option of making pedagogical processes more flexible in students from the articulation of web applications to continue with the educational service. The present study aimed to analyze the integration of ICT tools as a didactic strategy in collaborative learning in academic training spaces as part of the rethinking of teaching. For the research work, the mixed approach was used. The instrument used was the questionnaire, which allowed diagnosing the management and use of ICT tools to be able to respond to the individual and collective needs raised by educators and the directors of educational institutions. In the results obtained, it was evidenced that the educator had to assume a new role mediated by ICTs in the face of mandatory preventive isolation. Another finding was the strengthening of the use of ICT in the virtual context of the classes generated by the educators, given a new impetus to the training process based on innovative significant experiences. It is concluded that the pandemic generated a disruption in the educational system, for this reason, the directives of educational institutions should have as effective learning alternatives the virtual education model as an added value to their educational proposal in response to the new post-COVID context -19 .

Keywords: ICT tools, COVID-19, didactic strategy, educational innovation. 
La Educación en Tiempo del Covid-19 Herramientas TIC: El Nuevo Rol Docente en el Fortalecimiento del Proceso Enseñanza Aprendizaje de las Prácticas Educativa Innovadoras.
El mundo tiene hoy un gran desafío a causa de la pandemia de COVID-19, la Organización Mundial de la Salud, (OMS), ha declarado al virus como pandemia por su velocidad de propagación y por su alta escala de contagio, en consecuencia, en la mayoría de los países del mundo los gobiernos tomaron medidas de cerrar las instituciones escolares, con el fin de evitar el contagio en este sector de la población y como estrategia para contener la propagación del virus. De acuerdo con el informe presentado por la UNESCO en el mes de abril del 2020, el impacto inmediato del COVID-19 ha afectado alrededor del $70 \%$ de la población estudiantil del mundo.

En concordancia con lo anterior, en el contexto colombiano, y siguiendo las recomendaciones dadas por la OMS, el Ministerio de Educacional Nacional, (MEN) con el aval del gobierno central, emitió las orientaciones a las secretarías de educación, del territorio nacional, para dar cumplimiento del aislamiento preventivo obligatorio y poder mitigar la propagación del virus en las instituciones educativas, estableció la flexibilidad curricular con el fin de poder seguir brindando el apoyo al proceso de educativo y garantizar la prestación del servicio a los educandos.

Al mismo tiempo, el anuncio dado por el MEN tomó por sorpresa a la comunidad educativa de todos los establecimientos públicos y privados. Ante este hecho, los rectores y los docentes no estaban listos para desarrollar su propuesta educativa en el contexto del modelo a distancia. De acuerdo con el estudio realizado por el Laboratorio de Economía de Educación (LEE) de la Universidad Javeriana, el 96\% de los colegios públicos en Colombia no están preparados para implementar clases virtuales, el estudio también muestra, que más de la mitad de los estudiantes de colegios en el país no tienen computador e internet en sus hogares. Solo un porcentaje de colegios privados con plataforma virtual podrán seguir dando el servicio educativo en modalidad virtual.

Ante el nuevo contexto, el desafío es ahora para los educadores, los cuales, en su mayoría, son inmigrantes digitales, en consecuencia, "se debe buscar la forma de poder enseñar y conectar a los estudiantes con el propio proceso de aprendizaje" (Prensky 2010), por esta razón, se debe integrar las Tecnologías de la Información y la Comunicación (TIC) a los procesos formativos, sin embargo, hay que tener presente que la mayoría de los educadores, no tienen los suficientes conocimientos técnicos para el manejo de las tecnologías más habituales en las aulas, o simplemente no disponen de información sobre los beneficios que les pueden ofrecer en los procesos educativos. Por ende, los procesos de formación en los educadores enfocado a la integración de las TIC en el aula deben ser capaz de generar competencias tanto en los aspectos técnicos, como formativos y metodológicos de estas nuevas herramientas, ya que sin esa combinación las posibilidades de la articulación de las tecnologías se ven notablemente imperceptibles, (Rodríguez \& Pozuelos, 2009).

La incursión de las nuevas tecnologías en los contextos cotidianos ha hecho que estén presentes en las diferentes esferas de la sociedad. De acuerdo con el informe presentado por la UNESCO en el 2015 sobre el enfoque estratégico de las TIC en la educación en América Latina y el Caribe, demanda que el sistema de educacional, en los países de la región, requiere una actualización de las prácticas educativas al igual que los contenidos, los cuales deberían estar acordes a los avances de la nueva sociedad del conocimiento.

Es importante destacar que la irrupción de las TIC en los contextos escolares pone en evidencia lo importante de una nueva definición de roles, especialmente, para los estudiantes y educadores. Los primeros, gracias a estas nuevas herramientas, pueden adquirir mayor autonomía y responsabilidad en el proceso de aprendizaje, lo que obliga al educador a salir de su rol clásico como única fuente de conocimiento. Para Lugo (2010) manifiesta que "esto genera incertidumbres, tensiones y temores; realidad que obliga a una readecuación creativa en las instituciones escolares". Por lo tanto, para la nueva modalidad virtual en educación, el educador no sólo debe ser capaz de aplicar las tecnologías al servicio de la educación, sino también, debe tener las habilidades al igual que destrezas para diseñar nuevos escenarios educativos donde los estudiantes puedan aprender a moverse y poder participar en el espacio telemático (Echeverría, 2000).

El objetivo planteado para el presente estudio de investigación fue, analizar la integración de las herramientas TIC como estrategia didáctica en el proceso de aprendizaje colaborativo en los espacios de formación académica como parte del nuevo rol docente en las instituciones educativas Jardín Infantil San Gabriel Arcángel y colegio Sandra Catalina en la 
La Educación en Tiempo del Covid-19 Herramientas TIC: El Nuevo Rol Docente en el Fortalecimiento del Proceso Enseñanza Aprendizaje de las Prácticas Educativa Innovadoras. ciudad de Bogotá a raíz del aislamiento preventivo obligatorio.

\section{Herramientas TIC}

Las TIC, sigue generando un alto impacto al interior de la sociedad del conocimiento con respecto a la forma y al contenido, el efecto ha sido multiplicador más en el campo de la educación. Las TIC, ahora son más amigables, accesibles, y adaptables en los contextos educativos. Esta incorporación, en los espacios de formación pedagógica es con el propósito de hacer la transición de lo tradicional hacia un aprendizaje más constructivo por parte de los educadores. Parra (2012), menciona que "uno de los espacios donde el proceso tecnológico ha influenciado mayoritariamente es en los contextos educativos, naturalmente, ha sido en el oficio del maestro, llegando a formar parte de su cotidianidad escolar" (p.145).

En ese sentido, es importante determinar que "la capacidad de transformación y mejora de la educación de las TIC debe entenderse como un potencial que puede o no hacerse realidad, (...) en función del contexto en el que estas tecnologías son efectivamente utilizadas" (Coll, 2008, p.15) por esta razón, en el contexto actual, el nuevo rol docente, es poder articular las herramientas TIC que hay en su contexto educativo para poder generar nuevos procesos disruptivos en el aprendizaje, los cuales sean significativo para los estudiantes.

Como lo indica Schiavo (2007) "el principal uso que se les da a las TIC es las de poderlas utilizar como una herramienta que acelera procesos y por tal motivo disminuir el tiempo de dedicación a los mismos" (p. 91). En este sentido. los educadores con el apoyo tecnológico en el proceso de enseñanzaaprendizaje, están contribuyendo a estimular nuevos procesos de aprendizaje, donde los estudiantes son los actores principales de su proceso de formación y aprehensión de los nuevos saberes. Delgado (2020) señala que "actualmente estamos viviendo una época de cambios, en la cual tanto niños como adolescentes son los que utilizan más tiempo enlazados con las nuevas tecnologías", por ende, los docentes no pueden desconocer los contextos de sus estudiantes.

Ante el aislamiento preventivo obligatorio que acobija a las instituciones escolares, se hacen necesario que los educadores implementen el uso de herramientas TIC como estrategia didáctica en el proceso de aprendizaje con los estudiantes. De acuerdo con lo planteado por Arrieta et al. (2010) algunos de los recursos TIC que se pueden usar son: Videoconferencia, Buscadores, Software especializado, Espacios virtuales de comunicación (foros, debates,), correo electrónico, chats y mensajería instantánea, materiales didácticos multimedia (en línea o local), listas de discusión/distribución, pizarra electrónica, Hardware (impresora, escáner, cámara digital, etc.).

Para Barráez (2020) "la integración de las TIC en la educación ha abierto grandes posibilidades para enriquecer los procesos de enseñanza y de aprendizaje en los espacios virtuales", en este sentido, los docentes han tenido que afrontar los nuevos desafíos en la articulación de las herramientas TIC para llevar a cabo las clases en modalidad virtual $\mathrm{y}$, por ende, contribuir a seguir cerrando la brecha tecnológica en el momento del uso de las herramientas tecnológicas.

En el nuevo escenario para los colegios, las directrices acerca de la virtualidad, hay que tener presente una nueva variable en el proceso de enseñanza aprendizaje, la pantalla del computador o dispositivo móvil, los profesores se ven forzados a modificar su forma de trabajo hacia un encuentro con las nuevas reglas tecnológicas, convirtiéndose en el organizador entre los estudiantes y los objetos de conocimiento, estimulando permanentemente la iniciativa y en el aprendizaje activo con creación, comunicación y participación mediado por la articulación de las herramientas TIC, como lo menciona Coll (2008) "las expectativas en el uso de las TIC para mejorar del proceso formativo son muy elevadas y la realidad se ha quedado muy corta" ( $\mathrm{p}$. 17).

\section{Estrategia Didáctica}

El proceso de la enseñanza requiere una organización y planificación por parte del educador, en este orden de ideas, la planeación de las actividades pedagógicas requiere pensar en la metodología y los recursos más indicados para que los contenidos programáticos se puedan desarrollar en los estudiantes de una manera apropiada.

En este sentido, el proceso de la enseñanza emplea estrategias didácticas como parte de su organización en el proceso. Para referirse al concepto 
La Educación en Tiempo del Covid-19 Herramientas TIC: El Nuevo Rol Docente en el Fortalecimiento del Proceso Enseñanza Aprendizaje de las Prácticas Educativa Innovadoras. de didáctica, es necesario mencionar la etimología del término, Amós (2000) menciona que "el término didáctica proviene del griego didasticós, que significa el que enseña y concierne a la instrucción" (p.10), por consiguiente, las estrategias didácticas son herramientas útiles que ayudan al educador a comunicar los contenidos planeados y para hacerlos más amigables para la comprensión de los estudiantes.

De acuerdo con Tébar (2003), la estrategia didáctica consiste en "los procedimientos que el agente de enseñanza utiliza en forma reflexiva y flexible para promover el logro de aprendizajes significativos en los estudiantes" (p.7). Cabe resaltar que existe otra aproximación para definir una estrategia didáctica, para Tobón (2010) las estrategias didácticas son "un conjunto de acciones que se proyectan y se ponen en marcha de forma ordenada para alcanzar un determinado propósito" (p. 90), por ello, en el campo pedagógico específica que se trata de un "plan de acción que pone en marcha el docente para lograr los aprendizajes" Tobón (2010), es el plan de acción que requiere que el educador implemente en el contexto actual de la pandemia del COVID-19.

Por consiguiente, la estrategia didáctica de enseñanza donde se vincula las herramientas tecnológicas le permite al educador identificar sus diferentes habilidades para ser usadas en el aula. Estas estrategias, son importante si su aplicación está correlacionada entre las herramientas TIC y la propuesta académica, adicionalmente, esto permitirá encontrar un sentido didáctico de la tecnología donde dará una incorporación realmente significativa al proceso formativo.

\section{Innovación Educativa}

Con el paso del tiempo, se ha generado una efervescencia mediática acerca de la conceptualización de innovación educativa. Son varios los autores que han realizado aportes sobre la definición acerca de este tema. Imbernón (1996) afirma que "la innovación educativa es la actitud y el proceso de indagación de nuevas ideas, propuestas y aportaciones, efectuadas de manera colectiva, para la solución de situaciones problemáticas de la práctica educativa" (p.64).

En el contexto educativo nunca se parte de cero; siempre hay una experiencia que se ha recorrido. El proceso de innovación está directamente relacionado con obtener nuevos conocimientos y con la implementación de procesos creativos. Desde una perspectiva más amplia. Carbonell (2002), define la innovación como "una serie de intervenciones, decisiones y procesos, con cierto grado de intencionalidad y sistematización que tratan de modificar actitudes, ideas, culturas, contenidos, modelos y prácticas pedagógicas" (p.103).

Es innegable no incluir los conceptos de cambio y mejora cuando se habla de innovación educativa. Para Sein-Echaluce (2016) "La innovación educativa es la aplicación de una idea que produce cambio planificado en procesos, servicios o productos que generan mejora en los objetivos formativos" (p. 596). Es importante que los educadores puedan facilitar a los educandos las innovaciones propuestas en el proceso educativo con el fin de poder identificar el impacto generado como innovación educativa al proceso propuesto. Con el arribo de las TIC a los contextos escolares, se ha dado un nuevo impulso a los métodos pedagógicos, propiciando en el sistema escolar la búsqueda de nuevos caminos innovadores en el proceso formativo.

Por lo tanto, son los educadores del siglo XXI, en su nuevo rol docente, los que están llamados a liderar, a partir de experiencias innovadoras, el desarrollo de las diferentes capacidades en los estudiantes, como son: aprender a trabajar en equipo, empatía, creatividad, comunicación, inclusión, entre otras, apoyadas desde las herramientas TIC. De acuerdo con lo planteado por Hillmayr (2020) en el estudio de investigación realizado sobre el uso de las herramientas digitales en el área de matemáticas, se concluye que "el uso de las herramientas digitales tuvo un efecto positivo en los resultados de aprendizaje de los estudiantes". De acuerdo con lo anterior, cualquier propuesta que implique cambio y mejora utilizando las TIC se constituye como una innovación al proceso de enseñanza aprendizaje.

\section{Metodología}

Para el desarrollo del presente trabajo de investigación se utilizó el enfoque mixto, de acuerdo con lo planteado Bryman (2006) con la mirada y el empleo de los métodos cualitativo y cuantitativo se aplicó en las instituciones educativas objeto del 
La Educación en Tiempo del Covid-19 Herramientas TIC: El Nuevo Rol Docente en el Fortalecimiento del Proceso Enseñanza Aprendizaje de las Prácticas Educativa Innovadoras. estudio, las muestras fueron a directivas, docentes incluyendo a padres de familia de los niveles educativos de preescolar y primaria en el Liceo Sandra Catalina y Jardín infantil San Gabriel Arcángel de la ciudad de Bogotá.

Para el estudio de la investigación, se utilizó el instrumento de la encuesta para identificar el impacto del COVID- 19 en el proceso pedagógico, también incluyó el uso de las TIC en el proceso educativo en las instituciones educativas objeto de estudio, posteriormente, se realizó estudios estadísticos de la información recolectada. El estudio bibliográfico permitió la construcción del marco teórico para definir la estructura en la investigación, valorando del estado del arte relacionado con el uso de las TIC en el aprendizaje.

\section{Población}

La población objeto de estudio está conformada por: directivas, docentes y padres de familia pertenecientes a los niveles educativos de básica primaria de los colegios de Sandra Catalina y Jardín infantil San Gabriel Arcángel en la ciudad de Bogotá.

\section{Tabla 1}

\section{Población encuestada en los contextos escolares}

\begin{tabular}{cc}
\hline Estratos & Total de la población \\
\hline Directivas & 05 \\
\hline Docentes & 25 \\
\hline $\begin{array}{c}\text { Padres de familia } \\
\text { Total }\end{array}$ & 99 \\
\hline
\end{tabular}

Nota. Esta tabla muestra la población que participó en el estudio de la investigación en los dos escenarios educativos, elaboración propia.

\section{Resultados}

\section{Figura 1}

Docentes: ¿La práctica docente en su contexto educativo qué tanto se ha visto impactado por el COVID-19?

¿La práctica docente en su contexto educativo qué tanto se ha visto impactado por el COVID-19?

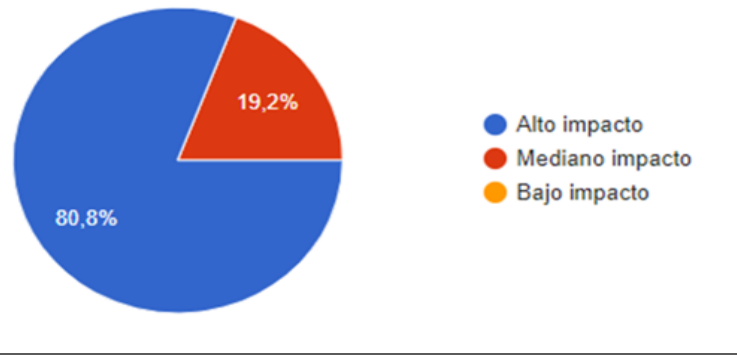

Nota. En los docentes encuestados, la mayoría manifiestan que la su práctica docente ha tenido un alto impacto por causa de la pandemia del COVID-19, elaboración propia.

\section{Figura 2}

Docentes: En una escala del 1 al 5, siendo 1 el más bajo y 5 el más alto, ¿Cree que el proceso de enseñanza aprendizaje de su clase con los estudiantes se ha visto afectada por el COVID-19?

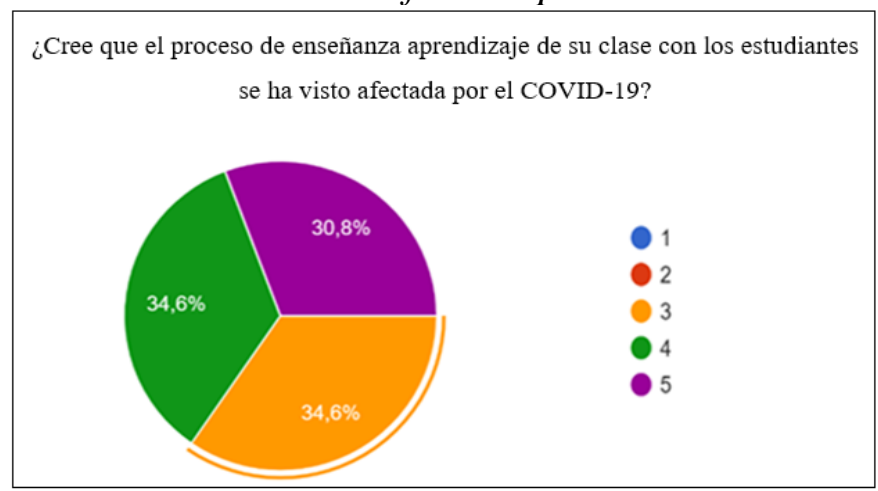

Nota. De la población docente estudiada el $34.6 \%$ considera que el proceso de enseñanza aprendizaje de las clases con los estudiantes se ha visto afectada por el COVID-19, elaboración propia.

\section{Figura 3}

Docente: ¿Qué tan difícil ha sido adaptarse al nuevo rol docente en los procesos enseñanza - aprendizaje en su contexto educativo?

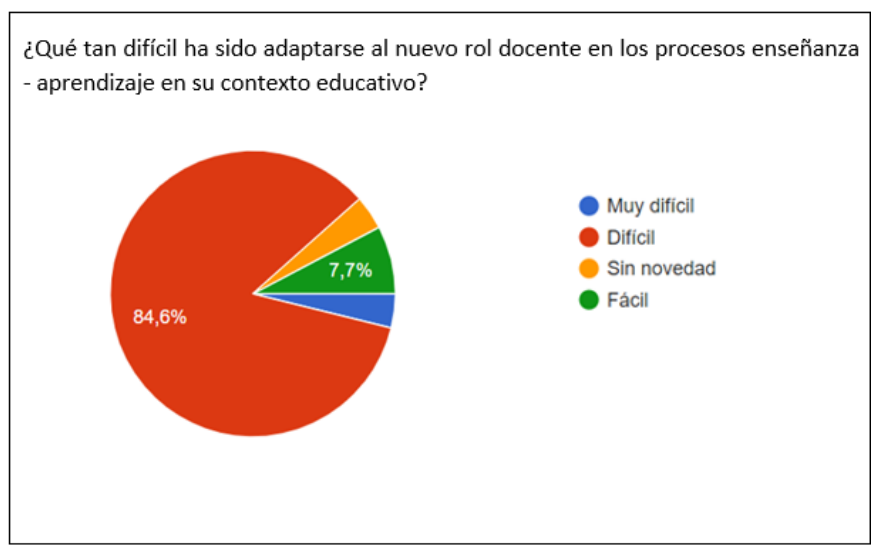

Nota. De la población docente estudiada, la mayoría considera ha sido difícil adaptarse en los procesos de enseñanza aprendizaje en su contexto educativo, elaboración propia. 
La Educación en Tiempo del Covid-19 Herramientas TIC: El Nuevo Rol Docente en el Fortalecimiento del Proceso Enseñanza Aprendizaje de las Prácticas Educativa Innovadoras.

\section{Figura 4}

Docente: Valore el dominio real de sus habilidades y competencias que tiene en el manejo de las herramientas TIC.

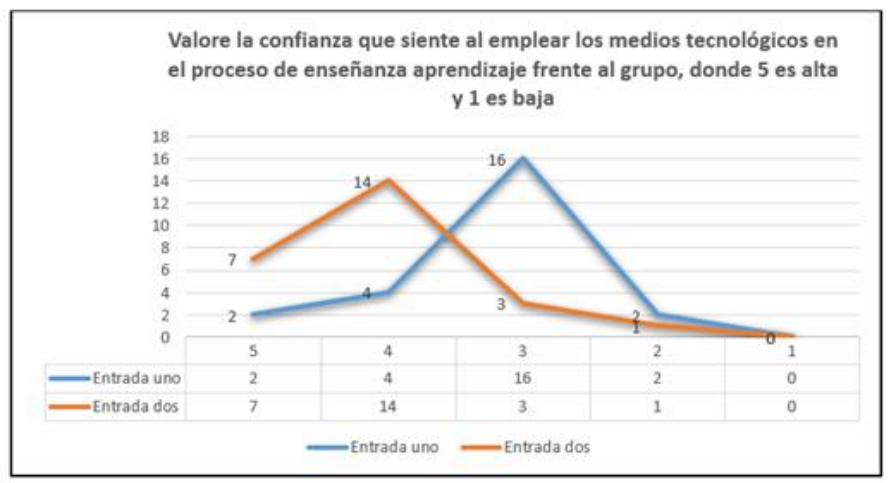

Nota. De la población docente estudiada, en la primera entrada se evidencia que doce docentes consideran insuficiente en el manejo de sus habilidades y competencias en el manejo de las herramientas TIC. En la segunda entrada, y después del acompañamiento realizado durante la investigación, solo un docente considera que sigue siendo insuficiente su dominio en el manejo de sus habilidades frente al manejo de las herramientas TIC, elaboración propia.

\section{Figura 5}

Docente: Valore la confianza que siente al emplear los medios tecnológicos en el proceso de enseñanza aprendizaje frente al grupo, donde 5 es alta y 1 es baja.

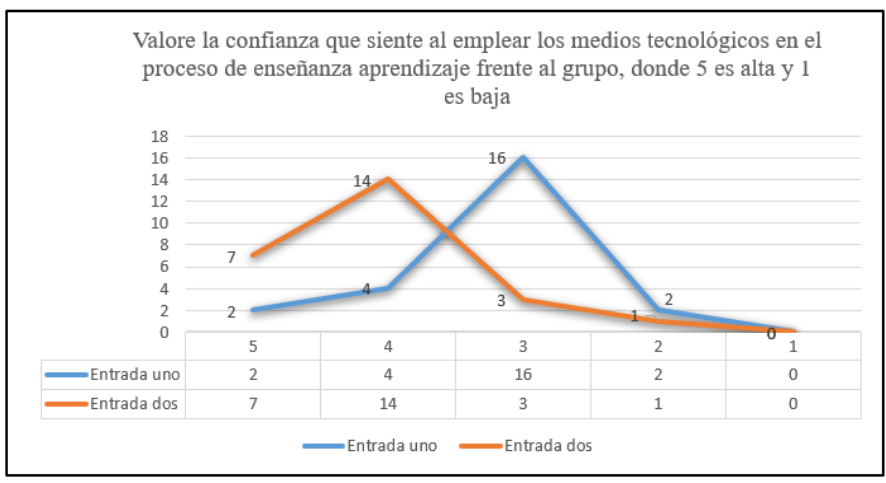

Nota. De acuerdo con los docentes encuestados, en la primera entrada se evidencia que dieciséis docentes consideran media la confianza que sienten al emplear los medios tecnológicos en el proceso de enseñanza aprendizaje frente al grupo. En la segunda entrada, y después del acompañamiento realizado durante el estudio, solo tres docentes consideran que su confianza sigue siendo media frente al ítem número 3 , elaboración propia.

\section{Figura 6}

Directivas: ¿Cómo directivo piensa que los entornos virtuales pueden ser hoy un medio eficaz para el proceso de enseñanza aprendizaje en su comunidad académica?
¿Cómo directivo piensa que los entornos virtuales pueden ser hoy un medio eficaz para el proceso de enseñanza aprendizaje en su comunidad académica?

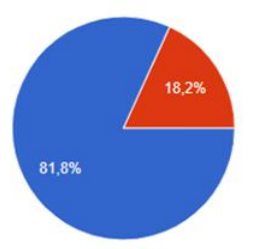

- Si

Nota. De acuerdo las directivas encuestadas, la mayoría considera que los entornos virtuales pueden ser hoy un medio eficaz para el proceso de enseñanza aprendizaje en su comunidad académica, elaboración propia.

\section{Figura 7}

Padres de Familia: Como padre de familia considera que las nuevas tecnologías a las que su hijo (a) tienen acceso generan.

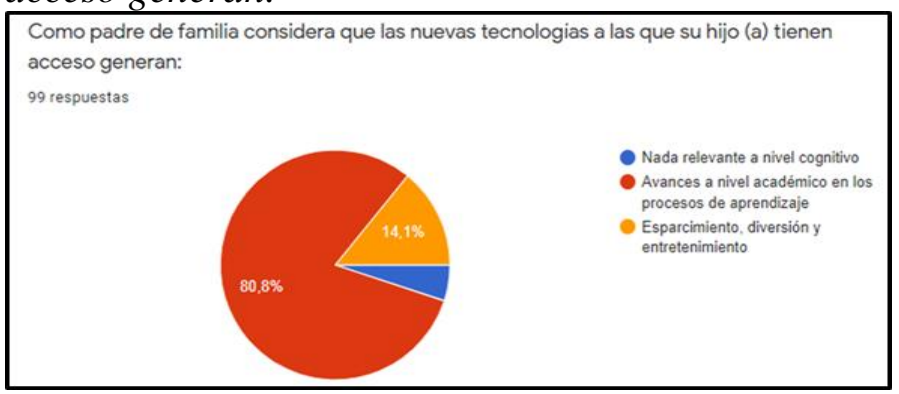

Nota. De acuerdo con los padres de familia encuestados en los dos contextos educativos, la mayoría considera que las nuevas tecnologías son un avance en el proceso académico para el aprendizaje en sus hijos, elaboración propia.

\section{Conclusiones}

Debido a situación presentada por la pandemia COVID-19, la UNESCO (2020) recalca que el 91\% de los estudiantes del mundo está siendo afectado. Por tal motivo el educador debe asumir un nuevo rol mediado por las TIC frente aislamiento preventivo obligatorio en las instituciones educativas. Como lo propone Freire (1997) "La educación no cambia al mundo: cambia a las personas que van a cambiar el mundo" (p. 60).

Sin duda, en el contexto actual de la modalidad virtual, los docentes han tenido que empezar un proceso de alfabetización funcional en el conocimiento y manejo de las herramientas TIC para poder ser implementadas como estrategias didácticas en el proceso ser implementadas como estrategias didácticas en el proceso formativo con sus estudiantes, por lo tanto, el propósito de integrar las TIC en los espacios de formación es mejorar el proceso de enseñanza aprendizaje, dando un uso 
La Educación en Tiempo del Covid-19 Herramientas TIC: El Nuevo Rol Docente en el Fortalecimiento del Proceso Enseñanza Aprendizaje de las Prácticas Educativa Innovadoras. oportuno y dinámico al proceso, por tal motivo, se hace necesario incentivar, a los educadores, en el uso de los recursos tecnológicos a través de capacitación en las competencias tecnológicas para docentes propuestas el Ministerio de la Educación Nacional, (MEN).

La articulación de las herramientas TIC en el contexto virtual de las clases generada por los educadores, ha dado un nuevo impulso a los procesos pedagógicos, creando experiencias significativas innovadoras para los estudiantes en su proceso de aprendizaje, claro está, evitando caer en la transferencia de información académica y procurar optar por modelos educativos flexibles (Villafuerte, 2019) para ratificar la formación humanizadora en el contexto del aislamiento preventivo obligatorio.

Durante el estudio de la investigación, en las instituciones escolares impactadas, se evidencia que el COVID-19 ha generado una disrupción en el sistema educativo, por tal razón, las directivas deben tener como alternativas de aprendizaje efectivo el modelo de educación en modalidad virtual como valor agregado a su propuesta educativa a partir del crecimiento de las competencias TIC en el desarrollo profesional docente.

\section{Referencias}

Amós, J. (2000). Didáctica Magna. México: Editorial Porrúa. http://www.terras.edu.ar/biblioteca/11/11DID_Fernandez_En guita_Unidad_1.pdf

Arrieta, R, Flores, M \& Martínez, O (2010). Articulación Pedagogía Tecnología: Un Medio para Mejorar las Competencias del Lenguaje y la Comunicación. Barranquilla, Educosta. (11), 143-146.

https://revistas.ugca.edu.co/index.php/sophia/article/view/347

Barráez, D. (2020). La educación a distancia en los procesos educativos: Contribuye significativamente al aprendizaje, Revista Tecnológica-Educativa Docentes 2.0: Vol. 8 Núm. 1 (2020): Ensayos

Bryman, A. (2006). Integrating quantitative and qualitative research: how is it done? Qualitative Research, (6) 97-113. https://doi.org/10.1177/1468794106058877

Carbonell, J. (2002). La aventura de innovar. El Cambio en la Escuela, Ediciones Morata. https://vdocuments.mx/carbonell-j-2006-laaventura-de-innovar-el-cambio-en-la-escuela.htm

Coll, C. (2008). Aprender y enseñar con las TIC: expectativas, realidad $y$ potencialidades. Boletín de la Institución Libre de Enseñanza, https://www.educ.ar/recursos/70819/aprender-y-ensenar-conlas-tic-expectativas-realidad-y-potencialidades
Delgado, J., García, C., Guaicha, K., \& Prado, M. (2020). La Webquest como herramienta didáctica para potenciar el pensamiento crítico en la formación de estudiantes universitarios. Revista Tecnológica-Educativa Docentes 2.0,9(1), 49-55. https://doi.org/10.37843/rted.v9i1.96

Echeverría J. (2000). Educación y tecnologías telemáticas. Revista Ibero Americana, 3-8. https://rieoei.org/historico/documentos/rie24a01.htm

Freire, P. (1997). Pedagogía de la Autonomía. Siglo XXI. (Original publicado en 1996). https://redclade.org/wpcontent/uploads/Pedagog\%C3\%ADa-de-laAutonom\%C3\%ADa.pdf

Hillmayr, D., Ziernwald, L., Reinhold, F., Hofer, S. I., \& Reiss, K. M. (2020). The potential of digital tools to enhance mathematics and science learning in secondary schools: A context-specific meta-analysis. Computers and Education, 153 doi: 10.1016/j.compedu.2020.103897

Imbernón, F. (1996). En busca del Discurso Educativo. Edit. Magisterio del Río de la Plata, Buenos Aires - Argentina. http://www.bnm.me.gov.ar/giga1/documentos/EL003277.pdf

Lugo, M.T. (2010). Las políticas TIC en la educación de América Latina. Tendencias y experiencias. Revista Fuentes, (10), 5268.

https://revistascientificas.us.es/index.php/fuentes/article/view/ $2587 / 0$

Ministerio de Educación Nacional. (2013) Competencias TIC para el desarrollo profesional docente. https://www.mineducacion.gov.co/1759/articles339097_archivo_pdf_competencias_tic.pdf

Parra, C. (2012). TIC, conocimiento, educación y competencias tecnológicas en la formación de maestros. Nómadas, 36, 145159. https://www.redalyc.org/pdf/1051/105124264010.pdf

Prensky, M. (2001). Digital natives, digital immigrants. On the Horizon, $9(5)$. https://www.marcprensky.com/writing/Prensky\%20$\% 20$ Digital\%20Natives, \%20Digital\%20Immigrants\%20$\% 20$ Part1.pdf

Rodríguez, F.P. \& Pozuelos Estrada, F.J. (2009). Aportaciones sobre el desarrollo de la formación del profesorado en los centros TIC. Estudios de casos. Revista de Medios y Educación, (35), 33-43. http://rabida.uhu.es/dspace/handle/10272/11693

Sein-Echaluce, M.L., Fidalgo-Blanco, A \& Alves, G (2016). Technology behaviors in education innovation. Computers in Human Behavior, In press. https://innovacioneducativa.wordpress.com/2018/01/17/unavision-emocional-de-la-innovacion-educativa/

Schiavo, E. (2007). Investigación científica y tecnológica en el campo de las TIC: ¿conocimientos técnicos, contextuales o transversales? Revista Iberoamericana de Ciencia, Tecnología y Sociedad - $\quad$ CTS, 3(9), 91-113. https://www.redalyc.org/articulo.oa?id=924/92430907

Tébar, L. (2003). El perfil del profesor mediador. Madrid: Santillana. http://www.cca.org.mx/apoyos/cu095/__m3.pdf 
La Educación en Tiempo del Covid-19 Herramientas

TIC: El Nuevo Rol Docente en el Fortalecimiento del

Proceso Enseñanza Aprendizaje de las Prácticas

Tobón, M. (2010). Formación integral y competencia, Pensamiento Complejo, diseño curricular y didáctica. Revista Interamericana de Educación de Adultos, 32(2),90-95. https://www.redalyc.org/articulo.oa?id=457545095007

Villafuerte, J. \& Demera, E. (2017). La asociación rural factor impulsor para el cambio de la matriz productiva. de En: B. Corral y F. Represa. Natura, Cultura y Desarrollo. Mar abierto.

https://issuu.com/marabiertouleam/docs/natura_cultura_de sarrollo

UNESCO (2020). Crisis-sensitive educational planning. Paris: UNESCO. http://www.iesalc.unesco.org/wpcontent/uploads/2020/04/COVID-19-Education-IssueNote-2.4-Planning-1.pdf 